\title{
Cardiac tamponade in association with anorexia nervosa: A case report and review of the literature
}

\author{
Jacqueline N. Kircher ${ }^{1}$, Matthew H. Park ${ }^{1}$, Michael K. Cheezum², \\ Edward A. Hulten², Jeffrey S. Kunz ${ }^{2}$, Mark Haigney ${ }^{3}$, J. Edwin Atwood ${ }^{2}$ \\ ${ }^{1}$ Department of Medicine, Walter Reed National Military Medical Center, Bethesda, MD, USA \\ ${ }^{2}$ Department of Medicine, Division of Cardiology, Walter Reed National \\ Military Medical Center, Bethesda, MD, USA \\ ${ }^{3}$ F. Edward Hebert School of Medicine, Uniformed Services University, Bethesda, MD, USA
}

\begin{abstract}
Anorexia nervosa (AN) is a complex psychiatric disorder that can have devastating cardiovascular complications. Its lesser-known association with pericardial effusion has been recently described in the literature. We present the case of a 45 year-old female who presented with a recurrent small bowel obstruction requiring lysis of adhesions and who was found to have a large pericardial effusion that progressed to cardiac tamponade necessitating surgical intervention. The patient had a body mass index of $14.8 \mathrm{~kg} / \mathrm{m}^{2}$ (i.e. $71 \%$ of ideal body weight) and a long-standing history of food aversion, extreme exercise habits, and weight obsession consistent with AN. To the best of our knowledge, this is the first case of AN-associated cardiac tamponade in the United States, and the first requiring surgical intervention. In conclusion, with this and current data regarding $A N$-associated pericardial effusions, we recommend a low threshold for performing pre-operative echocardiography for those in whom AN is suspected. (Cardiol J 2012; 19, 6: 635-638)
\end{abstract}

Key words: anorexia, pericardial effusion

\section{Introduction}

Anorexia nervosa (AN) is a serious and complex disorder characterized by fear of weight gain, caloric restriction, and a distorted self image that can have devastating health consequences, with cardiac complications constituting one of the major causes of death [1]. It is widely accepted that cardiac manifestations are commonly seen with this disorder, including electrocardiographic abnormalities, orthostatic hypotension, and poor myocardial contractility [1]. The lesser-known association with pericardial effusion has been increasingly described in the literature. We present a case of AN-associa- ted pericardial effusion progressing to cardiac tamponade in a young female with no previous cardiac history.

\section{Case report}

A 45 year-old female presented to the emergency department with a recurrent small bowel obstruction requiring lysis of adhesions and exploratory laparotomy. While undergoing induction of anesthesia with propofol, the patient became acutely hypotensive and bradycardiac, with associated non-sustained ventricular tachycardia. An electrocardiogram (ECG) revealed transient inferior

Address for correspondence: Michael Cheezum, MD, Cardiology Service, Department of Medicine, Walter Reed National Military Medical Center, 8901 Rockville Pike, Bethesda MD 20889, USA, tel: (301) 295-7836, fax: (888) 330-3498,

e-mail: michael.cheezum@us.army.mil

Received: 21.09.2011 Accepted: 26.09.2011 


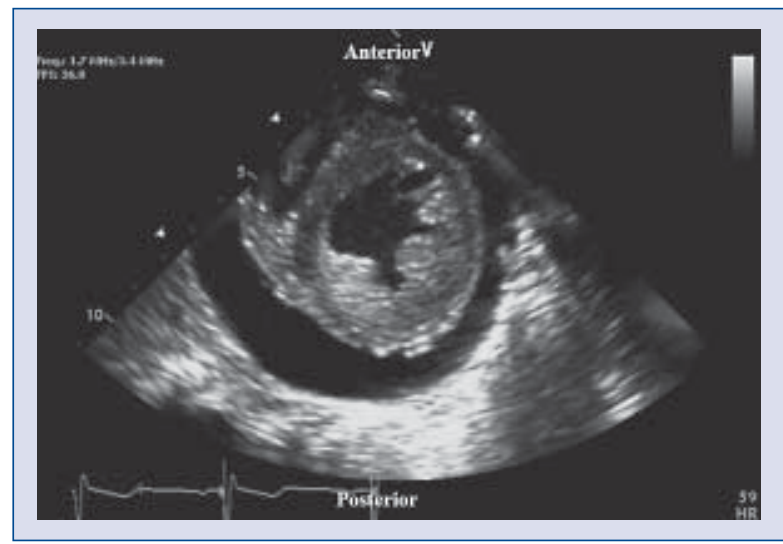

Figure 1. Parasternal short axis view of effusion; large, $2.5 \mathrm{~cm}$ in circumference, predominantly posterior pericardial effusion.

ST-depressions which resolved after initial fluid resuscitation, with subsequent cardiac enzyme elevations (peak $\mathrm{TnT}=0.22 \mathrm{ng} / \mathrm{mL}$, normal $<0.1$ ), suggestive of demand-related (type 2) myocardial infarction (MI). A transthoracic echocardiogram (TTE) was performed, which revealed a normal ejection fraction $(\mathrm{EF}=55-60 \%)$ with normal regional wall motion, and an incidental, large $(2.5 \mathrm{~cm}$ in circumference), predominantly posterior pericardial effusion with no initial echocardiographic evidence of tamponade (Fig. 1).

Over the next 24 hours, she became progressively hypotensive with narrowing pulse pressures, decreased urine output, and transient desaturations necessitating 2L oxygen via nasal cannula. Physical exam revealed jugular venous distention to $15 \mathrm{~cm}$ $\mathrm{H}_{2} \mathrm{O}$, and a pulsus paradoxus of $10 \mathrm{~mm} \mathrm{Hg}$. Repeat ECG demonstrated diffuse low voltage without electrical alternans. Follow-up TTE showed a persistent pericardial effusion with new, subtle late diastolic collapse of the right ventricular free wall (Fig. 2), enhanced ventricular interdependence with tricuspid and mitral inflow velocities of $26 \%$ and $18 \%$, respectively, and an increased inferior vena cava diameter ( $2.3 \mathrm{~cm}$ in M-mode) with decreased inspiratory collapse $(<50 \%)$, consistent with evolving tamponade.

Due to the predominantly posterior location of the effusion, she was taken to the operating room for a pericardial window. Pericardial fluid and tissue samples were sent for analysis.

Here, the patient admitted to having had bouts of food avoidance throughout her life, with extreme exercise habits that included daily 10 -mile runs. On admission, she had a body mass index (BMI) of $14.8 \mathrm{~kg} / \mathrm{m}^{2}$ (71\% of her ideal body weight), and chart

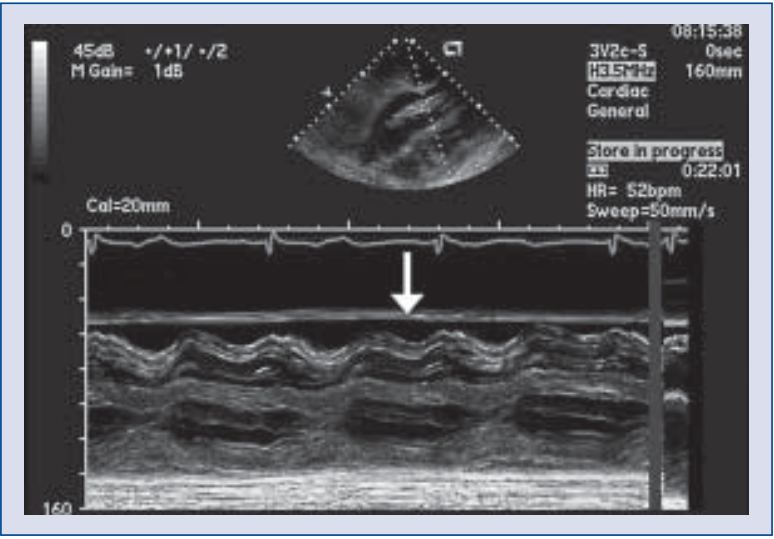

Figure 2. Two-dimensional (2D) subcostal view of the right ventricle $(R V)$; subcostal M-mode of $R V$ with $2 D$ inset demonstrating late diastolic collapse of the RV free wall (arrow).

review was notable for weights consistently below the DSM-IV cutoff for AN, defined as less than $85 \%$ of ideal body weight. Laboratory analysis revealed a history of mild hyponatremia $($ mean $=130 \mathrm{mmol} / \mathrm{L})$, hypoalbuminemia $(1.7 \mathrm{~g} / \mathrm{dL})$, and low prealbumin $(12.3 \mathrm{mg} / \mathrm{dL})$. Her pericardial fluid analysis and other labs were otherwise unremarkable, including a negative work-up for infectious or inflammatory causes with normal thyroid and renal function. Due to the patient's low to intermediate pre-test probability of having obstructive coronary artery disease (CAD), and the suspected demand nature of her MI, cardiac computed tomographic angiography was performed. This revealed no evidence of CAD. The patient recovered uneventfully from her pericardial window and is currently undergoing treatment for AN.

\section{Discussion}

We describe a rare case of cardiac tamponade as a complication of AN with significant hemodynamic compromise in the perioperative setting. Only one previous report has described AN-associated tamponade requiring pericardiocentesis [2]. A recent case series described three patients with AN-associated pericardial effusions, two of whom underwent elective pericardiocentesis to prevent tamponade [3]. To the best of our knowledge, this is the first described case of AN-associated cardiac tamponade in the United States, and the first requiring surgical intervention.

While tamponade is a very unusual complication of AN, pericardial effusions appear to be more common than previously recognized (Table 1 ). The association was first noted in 1983 in a series of four 


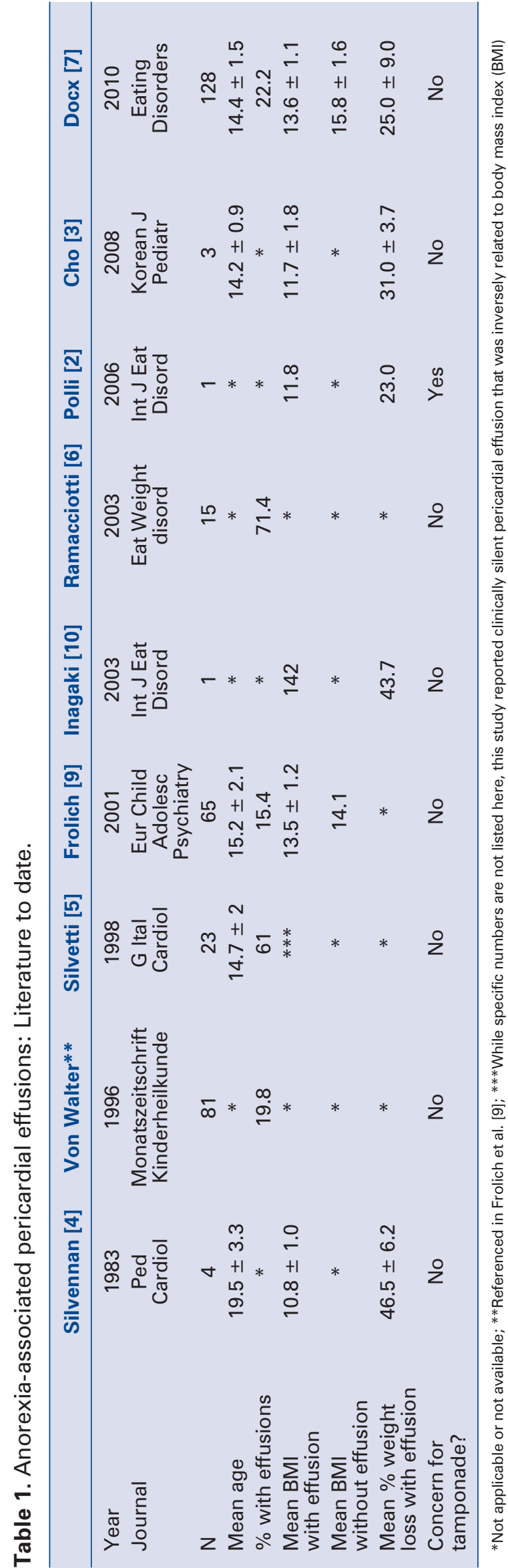

AN patients with pericardial effusions [4]. One study of echocardiographic findings in 23 asymptomatic AN patients found a mild to moderate pericardial effusion in $61 \%$ of patients; the presence of this finding, as well as a reduction in left ventricular thickness and mass, appeared to be inversely related to BMI, caloric intake, free T3 levels, and sodium concentrations [5]. Another study comparing echocardiography in a sample of $15 \mathrm{AN}$ patients compared to ten constitutionally thin controls of comparable age and activity levels found clinically silent pericardial effusions in $71.4 \%$ of the patients vs $10 \%$ of the controls $(\mathrm{p}<0.05)$, suggesting that this finding could be suggestive of early cardiovascular involvement with the disease [6]. While these two articles reported clinically silent pericardial effusions in the majority of AN patients, other studies have found $\mathrm{AN}$-associated effusions in only $15-22 \%$ of patients [7].

The pathophysiology behind the development of pericardial effusions with AN remains unclear. Malnutrition itself may promote effusions. Indeed, children with severe protein malnutrition in subSaharan Africa frequently manifest pericardial effusions in the absence of co-infection with TB or HIV [8]. In these patients, most pericardial effusions resolved with nutritional support only. Conversely, one study of AN patients with pericardial effusions noted a correlation between normalization of body mass and resolution of effusions, yet in those cases there did not appear to be a significant protein deficiency [9].

In the largest study to date, Docx et al. [7] performed echocardiograms in $128 \mathrm{AN}$ patients and found that $22 \%$ of patients had clinically silent effusions that significantly resolved with three months of re-feeding. Specific risk factors included BMI $\leq$ $\leq 13.5 \mathrm{~kg} / \mathrm{m}$, weight loss $\geq 25 \%$ and IGF-1 level $\leq$ $\leq 100 \mathrm{ng} / \mathrm{mL}$. They concluded that the presence of pericardial effusions are potentially due to the reduction in pericardial fat and myocardial muscle wasting, associated with more severe forms of starvation, and that the presence of pericardial effusions may be an indicator of AN disease severity [7].

\section{Conclusions}

Pericardial effusions are commonly seen in AN, and may progress to cardiac tamponade in rare cases. In view of the literature to date, and the wide prevalence of reported $\mathrm{AN}$-associated pericardial effusions, it is reasonable to consider preoperative echocardiography for patients in whom AN is suspected. 


\section{Disclosures}

The views expressed in this paper reflect the opinions of the authors only and not the official policy of the United States Army, United States Navy, Uniformed Services University, or the Department of Defense. This is a U.S. Government work. There are no restrictions on its use. There are no conflicts of interest, financial disclosures, grant support or writing assistance.

\section{References}

1. Katzman DK. Medical complications in adolescents with anorexia nervosa: A review of the literature. Int J Eat Disord, 2005; 37 (suppl.): S52-S59.

2. Polli N, Blengino S, Moro $\mathrm{M}$ et al. Pericardial effusion requiring pericardiocentesis in a girl with anorexia nervosa. Int J Eat Disord, 2006; 39: 609-611.
3. Cho YK, Yang SJ, Ma JS. Pericardial effusion in three cases of anorexia nervosa. Korean J Pediatrics, 2008; 51: 209-213.

4. Silverman JA, Krongrad E. Anorexia nervosa: A cause of pericardial effusion? Pediatric Cardiol, 1983; 4: 127.

5. Silvetti MS, Magnani M, Santilli A et al. The heart of anorexic adolescents. Gionale Cardiol, 1998; 28: 131.

6. Ramaciotti CE, Coli E, Biadi O et al. Silent pericardial effusion in a sample of anorexic patients. Eat Weight Disord, 2003; 8: 68-71.

7. Docx M, Gewillig M, Simons A et al. Pericardial effusions in adolescent girls with anorexia nervosa: Clinical course and risk factors. Eat Disord, 2010; 18: 218-225.

8. Ahmad S, Ellis J, Nesbitt A et al. Pericardial effusions in children with severe protein energy malnutrition resolve with therapeutic feeding: A prospective cohort study. Arch Dis Child, 2008; 93: 1033-1036.

9. Frolich J, von Gontard A, Lehmkuhl G et al. Pericardial effusions in anorexia nervosa. Eur Child Adolesc Psychiatry, 2001; 10: 54.

10. Inagaki $\mathrm{T}$, Yamamoto $\mathrm{M}$, Tsubouchi $\mathrm{K}$ et al. Echocardiographic investigation of pericardial effusion in a case of anorexia nervosa. Int J Eat Disord, 2003; 33: 364-366. 